\title{
Caractérisation de jets d'agrégats d'argon pour l'étude de l'interaction laser-agrégats
}

\author{
F. Blasco, T. Caillaud, F. Dorchies, C. Stenz, A.S. Boldarev ${ }^{1}$ et V.A. Gasilov ${ }^{1}$ \\ Centre Lasers Intenses et Applications, UMR 5107 du CNRS, Université de Bordeaux I, France \\ ${ }^{1}$ Institute of Mathematical Modeling, Russian Academy of Sciences, Russie
}

\begin{abstract}
Résumé : Argon clusters jets were produced at the output of supersonic nozzles and used as targets for intense laser - clusters interaction experiments. They were fully characterized combining two optical diagnostics (Mach-Zehnder interferometry and Rayleigh Scattering) with the support of a numerical simulation. This study was performed with Laval and conical nozzles. Homogeneous clusters size profiles were observed at the output of the conical nozzle. Clusters mean radius was measured in the range from 200 to $300 \AA$, increasing with the input Argon pressure varying from 20 to 60 bars.
\end{abstract}

\section{INTRODUCTION}

L'interaction d'une impulsion laser intense avec des agrégats nanométriques fait, depuis quelques années, l'objet de nombreux travaux de recherche[1]. Ces agrégats d'atomes peuvent être obtenus par condensation spontanée d'un gaz rare lors de sa détente dans une buse supersonique. Ces agrégats présentent localement une densité proche de celle du solide susceptible d'induire une absorption collisionnelle plus élevée qu'avec une cible gazeuse. D'autre part, la quantité de matière chauffée par l'impulsion laser étant confinée aux seuls agrégats, les débris produits après l'interaction sont moins nombreux qu'avec une cible solide. Plusieurs expériences ont ainsi montré le potentiel de cette cible pour la production de particules énergétiques (UV, X, ions, neutrons, ...) à haute cadence et sans débris[2]. La compréhension des mécanismes mis en jeu nécessite une caractérisation précise des agrégats, notamment de leur taille qui est sans aucun doute un paramètre clé de l'interaction. Dans de nombreuses expériences déjà réalisées, les agrégats étaient obtenus à la sortie d'un dispositif classique comprenant une buse suivie d'une série d'écorceur. La taille des agrégats était déterminée à partir des lois empiriques obtenues, dans cette même géométrie, par Hagena à l'aide d'un spectromètre de masse[3]. L'optimisation des sources de rayonnement $\mathrm{X}$ et UV obtenues avec une cible composée d'agrégats entraîne les expérimentateurs à focaliser l'impulsion laser dans le jet de gaz directement à la sortie de la buse, où le nombre d'agrégats attendus est plus important. Le travail dans cette géométrie ne permet plus d'utiliser les formules d'Hagena. Il devient nécessaire de développer d'autres outils pour déterminer la taille et la répartition spatiale des agrégats dans le jet. Dans cet article, nous présentons un tel outil basé sur deux différents diagnostics optiques (interférométrie Mach-Zehnder et diffusion Rayleigh) et supportée par une simulation numérique rendant compte de la détente du gaz dans la buse ainsi que de la formation des agrégats. Cette étude a été réalisée en considérant l'écoulement d'Argon dans deux types de buse: une buse Laval et une buse conique.

\section{DISPOSITIF EXPERIMENTAL}

\subsection{Paramètres pour décrire le jet d'agrégats}

Le mélange formé par le gaz et les agrégats en sortie d'une buse peut être décrit par les trois paramètres suivants: a) la densité atomique totale $n_{\text {tot }}$ définie comme la quantité totale d'atomes incluant les atomes sous phase gazeuse et les atomes agrégés; b) le degré de condensation $\eta$ défini comme le rapport du nombre d'atomes sous forme agrégé sur le nombre total d'atomes; c) le rayon moyen $R$ des agrégats. Ces trois quantités sont des données locales dont il faut déterminer la répartition spatiale transverse et le long de l'axe de l'écoulement en sortie de buse. Dès qu'il contient 1000 atomes, la structure de l'agrégat est de type Cubique-Face-Centrée avec un paramètre de maille de l'ordre de $5,3 \AA$ conduisant à l'expression $N \approx R^{3}(A) / 9$ pour le nombre moyen d'atomes $N$ par agrégats et à une densité atomique dans l'agrégat de $2,7.10^{22} \mathrm{~cm}^{-3}$. 


\subsection{Buses étudiées}

Deux buses ont été étudiées. La première est une buse de type Laval. Elle est constituée d'un col de $900 \mu \mathrm{m}$ de diamètre suivi d'un profil de tuyère parabolique. Elle débouche $13 \mathrm{~mm}$ plus loin avec une section de sortie de $2,5 \mathrm{~mm}$ de diamètre. La deuxième buse est conique. Le diamètre du col est de 600 $\mu \mathrm{m}$. Il est suivi par un profil de tuyère linéaire qui débouche $20,2 \mathrm{~mm}$ plus loin avec une section de sortie de $4 \mathrm{~mm}$ de diamètre. Ces deux buses sont à symétrie cylindrique et sont entièrement caractérisées par leur profil. La détente du gaz et la formation des agrégats sont par conséquent supposées à symétrie de révolution autour de l'axe des buses dans toute la suite de l'analyse. Cette détente est pulsée à l'aide d'une électrovanne. La pression en amont des buses a été variée et contrôlée par un manomètre.

\subsection{Intérférométre Mach-Zehnder}

L'écoulement en sortie de buse a été diagnostiqué à l'aide d'un interféromètre de type Mach-Zehnder (Figure 1). Ce dispositif utilisant un laser He-Ne est décrit dans une récente publication[4]. Il mesure la différence de phase entre un bras se propageant dans le vide et un autre traversant le jet de gaz contenant les agrégats. Le plan de la buse est imagé sur le détecteur, fournissant une résolution spatiale à deux dimensions de l'écoulement. La phase relative mesurée est le résultat de la somme cohérente, dans l'axe de propagation du laser, des champs des différents dipôles oscillants que constituent les atomes du jet. En ceci, tous les atomes contribuent à cette phase relative de la même manière, qu'ils soient agrégés ou restés sous forme gazeuse. La phase relative est par conséquent directement proportionnelle à la densité atomique totale $n_{t o t}$. Plus exactement, la phase relative $\Delta \phi$ est donnée par l'intégration suivant l'axe $z$ du laser de:

$\mathrm{d} \Delta \phi(z)=(2 \pi / \lambda),(\beta / 2) \cdot n_{t o t}(z) \mathrm{d} z$

l'A $\lambda$ est la longueur d'onde du laser et $\beta=2,1.10^{-23} \mathrm{~cm}^{3}$ est la constante tabulée de Gladstone-Dale de l'Argon.

Considérant cette relation et faisant l'hypothèse de symétrie de révolution, la répartition spatiale de $n_{t o t}$ est déduite, à l'aide d'une transformation d'Abel, dans les trois dimensions. La résolution spatiale de ce diagnostic est inférieure à la taille de l'interfrange (de l'ordre de $100 \mu \mathrm{m}$ ).
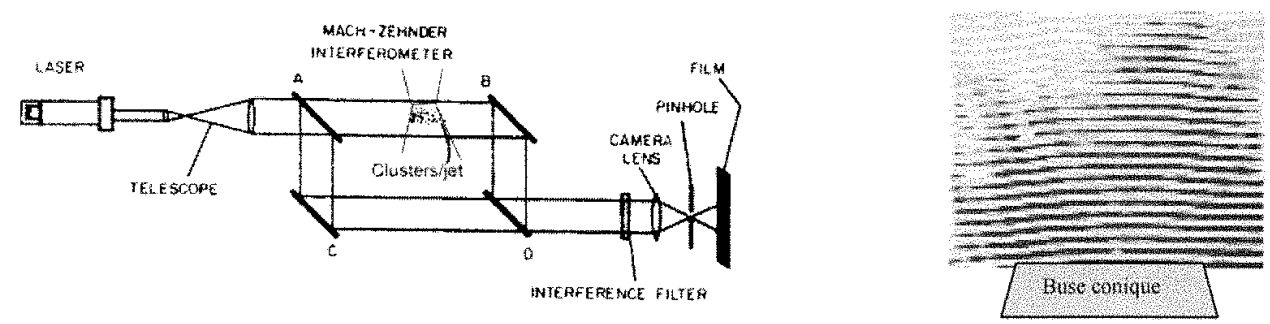

Figure 1: dispositif expérimental de l'interférométrie Mach-Zehnder et interférogramme du jet d'agrégats à 40 bars

\subsection{Diffusion Rayleigh}

Un diagnostic de diffusion Rayleigh a été mis en place au CELIA (Figure 2), de façon à caractérisation le phénomène d'agrégation dans les écoulements. Le dispositif est dans le principe très simple. Sa seule difficulté réside dans le soin extrême qu'il faut apporter pour réduire au maximum toute lumière parasite. La buse est installée de façon à engendrer un écoulement vertical. Un laser He-Ne de puissance moyenne stable se propage dans le plan horizontal. Il est focalisé dans le jet à une distance de la sortie de la buse que l'on peut ajuster. La lumière diffusée à $90^{\circ}$ est collectée par une lentille qui fait l'image du plan du jet sur une caméra CCD. L'angle solide de collection étant fixé par cette lentille, le signal enregistré sur un pixel de CCD est directement proportionnel à l'intensité $I_{d}$ de la lumière diffusée par l'élément de volume correspondant dans le jet à ce pixel. Cette dernière est reliée à l'intensité $I_{0}$ du laser par la relation suivante:

$$
I_{d} / I_{0}=C\left[n_{t o l}(1-\eta) \cdot \sigma_{a t}+n_{a g r} \cdot \sigma_{a g r}\right]
$$

Elle est la somme de la contribution des atomes isolés de la phase gazeuse et de celle des agrégats. $C$ est un facteur de proportion lié à la géométrie de la détection. $n_{a g r}$ désigne le nombre d'agrégats par élément de volume. $\sigma_{a t}$ et $\sigma_{a g r}$ sont respectivement les sections efficaces de diffusion Rayleigh d'un atome 
seul et d'un agrégat. Les agrégats considérés par cette étude sont de tailles très petites $(\leq 350 \AA)$ devant la longueur d'onde du laser $\left(6328 \AA\right.$ ). L'intensité diffusée à $90^{\circ}$ par $N$ atomes sous phase gazeuse est le résultat de la somme non-cohérente des champs diffusés par chacun des atomes. Elle vaut donc $N$ fois l'intensité diffusée par un seul atome. Si ces $N$ atomes sont agrégés sur une extension très petite devant la longueur d'onde laser, tous leurs champs diffusés s'ajoutent en phase. L'intensité totale diffusée devient alors $N^{2}$ fois l'intensité diffusée par un seul atome. Ceci s'écrit encore[5]:

$$
\sigma_{a g r}=N^{2} \sigma_{a r}
$$

En conservant rigoureusement la même géométrie (et donc le même facteur $C$ ), des mesures de calibration ont été effectuées dans l'enceinte remplie d'un gaz statique d'Argon dont la densité était contrôlée par un manomètre. Par la suite et en utilisant les valeurs de $n_{\text {fot }}$ obtenues avec le montage MachZehnder, nous avons normalisé les valeurs mesurées de l'intensité diffusée pour le jet d'agrégats par l'intensité que diffuse la même densité totale d'atomes sous phase gazeuse uniquement. Le résultat $M$ de cette normalisation s'écrit:

soit encore :

$$
\begin{array}{ll}
M=\left[(1-\eta)+\left(n_{a g r} . \sigma_{a g r}\right) /\left(n_{t o t} \cdot \sigma_{a t}\right)\right] & \\
M=[(1-\eta)+\eta \cdot N]=\eta \cdot N & \operatorname{car} N>\eta
\end{array}
$$

Ce diagnostic détermine donc le produit du nombre moyen $N$ d'atomes par agrégats par le taux de condensation $\eta$ dans le jet. 11 le fait avec une résolution spatiale dans les trois dimensions limitée le long de l'axe de propagation du laser par l'imagerie (de l'ordre de $10 \mu \mathrm{m}$ ) et dans les deux autres dimensions par la taille du laser He $\mathrm{Ne}$ focalisé (de l'ordre de $100 \mu \mathrm{m}$ ).
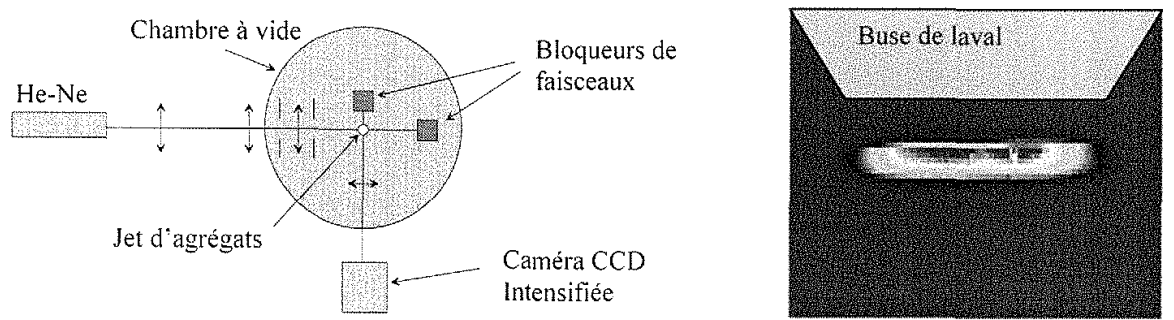

Figure 2 : Dispositif expérimental de diffusion Rayleigh et image de diffusion du jet d'agrégats à 40 bars.

\section{3 - Résultats expérimentaux}

Les expériences ont été effectuées avec des temps d'ouverture d'électrovanne de 20 ms. L'établissement d'un régime stable d'écoulement a été observé en moins de $2 \mathrm{~ms}$ après l'ouverture de l'électrovanne. La détermination de ce temps est entièrement limitée par la résolution temporelle apportée par un obturateur sur le faisceau He-Ne. La densité mesurée juste en sortie de buse suit pour chacune des deux buses étudiée, une loi linéaire avec la pression en amont de la buse. La figure 3 représente les profils de $n_{t o t}$ mesurés à $1,5 \mathrm{~mm}$ du plan de sortie de la buse conique, avec 40 bars d'Argon en amont. La buse conique présente un profil plus carré de $4 \mathrm{~mm}$ de diamètre à mi-hauteur. Dans le cas de la buse Laval, le profil est de type gaussien avec un diamètre à mi-hauteur de $2 \mathrm{~mm}$.

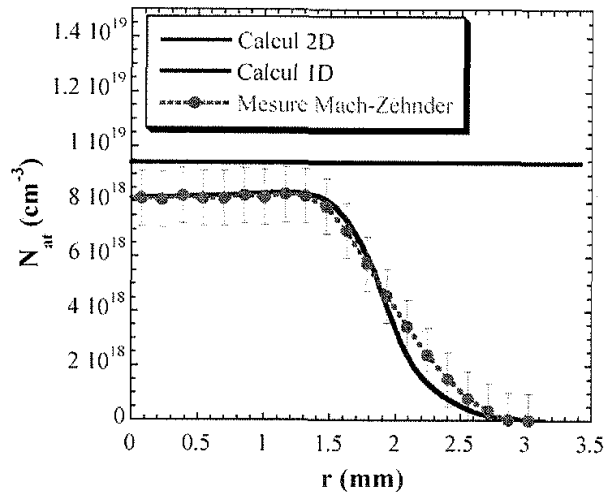

Figure 3 : Profil de densité de la buse conique

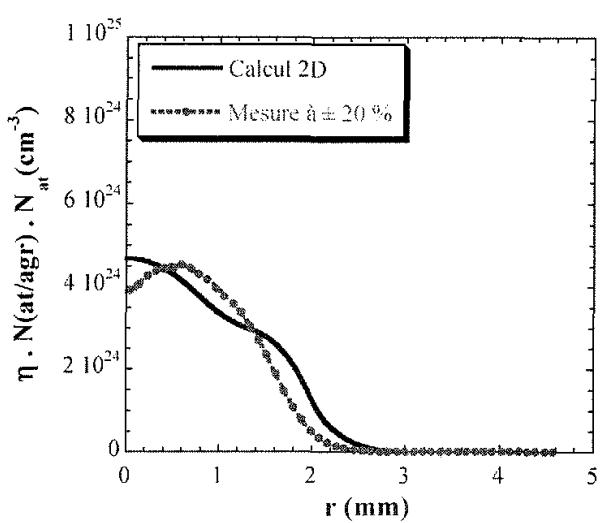

Figure 4 : Profil de $\eta . N$ de la buse conique 
La densité est maximale sur l'axe de buse et vaut, toujours pour 40 bars d'Argon en amont: $n_{t o l}=5.10^{19} \mathrm{~cm}^{-3}$ avec la buse Laval, $n_{t o t}=9.10^{18} \mathrm{~cm}^{-3}$ avec la buse conique.

Cette densité décroît au fur et à mesure que l'on s'éloigne du plan de sortie de la buse. Ceci s'accompagne d'un élargissement des profils. Sur la figure 4 sont reportés les profils obtenus pour la grandeur $M=\eta . N$ déduite des expériences de diffusion Rayleigh. Un profil très homogène est observé pour la buse conique. En revanche, il se présente sous une forme annulaire dans le cas de la buse Laval, indiquant l'absence d'agrégats sur l'axe de sortie de la buse. Il est à noter que le signal diffusé est plus important dans le cas de la buse conique que dans celui de la buse Laval, et de plusieurs ordres de grandeur.

\section{4 - Simulations numériques}

L'écoulement du gaz dans la buse, ainsi que la formation des agrégats ont fait l'objet d'un travail de simulation de la part d'une équipe de collaborateur ${ }^{6}$. L'écoulement est traité dans un régime non-turbulent et en négligeant les effets de viscosité. La formation et la croissance des agrégats est traitée dans cet écoulement en considérant un équilibre entre l'effet de la tension superficielle de l'agrégat supposé sphérique et son évaporation. Des simulations numériques ont été effectuées à l'aide de ce code, à partir des côtes exactes de nos deux buses. Un très bon accord est retrouvé dans la détermination des profils de densité atomique $\left(n_{i o t}\right)$ voir figure 3. D'autre part, les profils de la quantité $M=\eta \cdot N$ sont bien reproduits par le code (voir figure 4). Un profil annulaire est notamment observé dans ces simulations effectuées avec la buse Laval. Il est principalement la cause du profil du taux de condensation $\eta$, nul au centre et de l'ordre de quelques \% sur les bords. Dans le cas de la buse conique, on retrouve un profil homogène avec un taux de condensation $\eta$ de 23 à $28 \%$ pour des pressions en amont de 20 à 60 bars.

\section{5 - Discussion}

Les valeurs de $\eta$ sont estimées à partir des simulations qui reproduisent les deux mesures de façon très satisfaisante. Les tailles des agrégats sont par la suite déduites du calcul suivant $: R=(9 . N)^{1 / 3}=(9 . M / \eta)^{1 / 3}$ Il est à noter que l'impact d'une mauvaise évaluation de $\eta$ est réduit du fait de la puissance 1/3: un facteur 2 sur $\eta$ devient une erreur de $25 \%$ seulement. Les rayons d'agrégats ainsi déterminés au sortir de la buse varient entre 10 et $50 \AA$ lorsque la pression varie entre 20 et 60 bars en amont de la buse Laval. Le profil spatial de leur taille semble relativement homogène, le caractère annulaire des mesures de diffusion étant principalement induit par le profil de la concentration des agrégats. Celui-ci forme un anneau de $1,5 \mathrm{~mm}$ de diamètre. Ce diamètre augmente avec la distance à la sortie de la buse: il est doublé au bout de $5 \mathrm{~mm}$. La buse conique fournit un profil très homogène d'agrégats, à la fois en taille et en concentration sur un diamètre de $4 \mathrm{~mm}$. La distance à la sortie de la buse n'a pas dépassé $5 \mathrm{~mm}$ lors des mesures. A cette distance maximale, le diamètre du jet n'est guère modifié. Cependant, un affaissement au centre de $30 \%$ environ est observé au profit d'une croissance d'ailes sur les profils de gaz et d'agrégats. La taille des agrégats varie entre 200 et $300 \AA$, en association avec une pression en amont croissant de 20 à 60 bars.

\section{6 - Conclusion}

Nous avons mis en cuvre deux diagnostics optiques (interféromètre Mach-Zehnder et diffusion Rayleigh) afin de pleinement caractériser le mélange de gaz et d'agrégats à la sortie d'une buse supersonique. Cette étude a été menée sur deux types de buse: Laval et conique. Les mesures sont reproduites par une simulation numérique et indiquent l'obtention d'un profil très homogène d'agrégats avec la buse conique. Le rayon de ces agrégats peut être ajusté entre 200 et $300 \AA$, en contrôlant la pression en amont de la buse.

\section{Remerciements}

Ce travail a été en partie financé par le Fond Européen de Développement Economique Régional, le Conseil Régional d'Aquitaine et la Russian Foundation for Basic Research (projet $\mathrm{n}^{\circ}$ 00-01-00562).

\author{
Références \\ 1 T. Ditmire et al., Phys. Rev. A 53 (1996) 3379 \\ $2_{\mathrm{T}}$. Ditmire et al., Nature 398 (1999) 489 \\ 3 O.F. Hagena, Surf.Sci. 106 (1981) 101
}

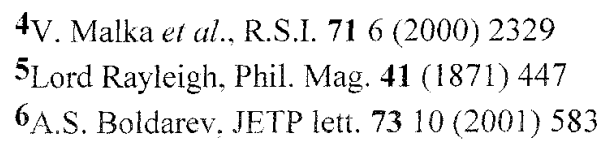

